\title{
Comparison Between Thick Blood Films, Quantitative Buffy Coat Technique and the ParaSight-F Dipstick in the Diagnosis of Malarial Infections
}

\author{
HAYTHAM A. ZAKAI, PhD \\ Department of Medical Technology Program, Faculty of Applied Medical Sciences \\ King Abdulaziz University, Jeddah, Saudi Arabia
}

\begin{abstract}
Three different laboratory methods for diagnosing malarial infections, especially with Plasmodium falciparum, were evaluated in this study using thirty infected samples (22 samples contained Plasmodium falciparum, 8 samples contained $P$. vivax.). These methods included stained thick blood films, Quantitative Buffy Coat system, and Dipstrip (ParaSight-F) immunoassay. Comparison among the three methods was done regarding the specificity to detect malaria parasites, the time required to perform the examination, and the advantages and disadvantages of each method. ParaSightF gave cross reactions with samples that contained high levels of rheumatoid factor (R.F.) and did not detect P. vivax. Quantitative Buffy Coat technique did not require technical skills but needed a long time to perform and species identification was very difficult. Thick blood films needed a longer time to be examined as well as special skills and experience, but it is still the method of choice since it can be applied in any laboratory or in the field with minimum equipments.
\end{abstract}

Keywords: Malaria, Plasmodium, Quantitative Buffy Coat system, Diagnosis, ParaSight-F

\section{Introduction}

Malaria is one of the most important parasitic diseases and is considered as one of the major factors contributing to the socio-economic conditions prevailing in developing

$\overline{\text { Correspondence \& }}$ reprint requests: Dr. Haytham A. Zakai

P.O. Box 80205, Jeddah 21589, Saudi Arabia

Accepted for publication: 09 November 2003. Received: 15 October 2000. 
countries. More than 200 million cases of malaria and at least one and a half million consequent deaths are estimated to occur annually. Over one-half of the world's population live in areas where malaria is endemic. Although formally found throughout most of the world, with seasonal outbreaks extending into temperate zones, malaria is, now, generally restricted to tropical and subtropical regions ${ }^{[1]}$.

The classical method for diagnosing malaria is by a microscope examination of the blood for malaria parasites. Other diagnostic methods, such as the Quantitative Buffy Coat (QBC) method, antibody detection by serological methods, DNA probes, have been and/or are being developed; but the microscopic examination of blood using thick and thin blood films remains the most common approach in the laboratory diagnosis ${ }^{[2,}$ $3]$.

QBC technique was used by several centers for the diagnosis of malaria infetions and is a reliable method for screening the population for malaria and for detetion of asymptomatic carriers $^{[4,5]}$. ParaSight-F, on the other hand, is used to diagnose Plasmodium fulciparum infections only and because of its high sensitivity to Plasmodium fulcipar$u m$, it is condisdered as the method of choice for screening for such an infection ${ }^{[6-8]}$.

The main objective of this study was to evaluate and to discuss the value of the thick blood film, Quantitative Buffy Coat technique, and the Parasight ${ }^{\mathrm{TM}}$ F Test Strip for the diagnosis of malaria specially infections with Plasmodium falciparum.

\section{Material and Methods}

Blood specimens with malaria parasites. Thirty infected blood samples were provided by the Hematology Laboratory of King Abdulaziz University Hospital (KAUH), Jeddah; the Parasitology Laboratory of King Saud Hospital, Jeddah; the Hematology Laboratory of King Fahd Hospital, Jeddah; Al Noor Hospital, Makkah; and the Hematology Laboratory of the Armed Forces Hospital, Jeddah. Some of the samples contained $P$. falciparum and others contained $P$. vivax all collected in EDTA anticoagulant. Ten known negative control blood samples were obtained from the Hematology Laboratory of KAUH and seven serum samples with high Rhomatoid Factor (R.F.) levels from different patients were provided by the Immunology Laboratory of KAUH. The average time required to process the samples by each test was estimated.

Thick blood film. The method described by Cheesbrough ${ }^{[2]}$ was used to prepare thick blood films. A drop of blood, collected in EDTA anticoagulant, was placed on a glass slide and spread around in a circle using the edge of another slide. Slides were then air-dried. Blood films were unfixed, or fixed by heat or absolute methanol, prior to staining. Giemsa stain was used for staining the thick blood films.

Parasight $^{\mathrm{TM}}$ F Dipstrips. Kits of Parasight ${ }^{\mathrm{TM}} \mathrm{F}$ dipstrips were kindly provided by 
the Al Saif Company, Jeddah. The test is a rapid test for the qualitative detection of the Histidine Rich Protein II (HRPII) antigen of P. falciparum directly from the whole blood without any instrumentation. It is commercially available by Becton Dickinson.

HRPII is a water-soluble protein released from parasitized erythrocytes ${ }^{[3]}$. It has been found in all natural isolates of P. falciparum and has been detected in plasma and urine as well as whole blood ${ }^{[4-6]}$.

Antibodies specific for $P$. falciparum HRPII were immobilized on a test strip. $P$. falciparum antigen is lysed whole blood binds to the antibody as the blood absorbs into the test strip. Detector particles containing a dye and coated with antibodies specific for $P$. falciparum absorb to the test strip and bind if the antigen is present. A solid pink line indicates a positive test. A pink procedural control dash and a white to light pink background will always be present if the test has been performed properly.

QBC (Quantitative Buffy Coat system). One kit of Acridine Orange coated tubes was kindly provided by the Al Saif Company, Jeddah. The principle behind the QBC system involves the collection of blood in special capillary tubes pre-coated with Acridine Orange (AO). The blood in the capillary tube is then mixed well. A float is inserted in the capillary tube and the tube is centrifuged. The buffy coat is then examined under a fluorescent microscope after placing the tube on a special microscope slide adaptor.

\section{Results}

The thick blood film, Parasight ${ }^{\mathrm{TM}}$ F Dipstrip immunoassay, and the Quantitative Buffy Coat technique were done on all the thirty infected blood samples and the ten negative control blood samples. All samples and controls were processed the same but only Parasight ${ }^{\mathrm{TM}}$ F Dipstrip immunoassay was done on the seven serum samples. Light microscopy was used in observing the thick blood films and a fluorescence microscopy used for QBC.

All thirty infected blood samples showed positive results with the stained thick blood films, eight identificated as infections with $P$. vivax and the rest as infections with $P$. falciparum. The negative control samples gave negative results. The average time required to process and examine the thick blood film was 19 minutes.

By using QBC technique, all the infected blood samples gave positive results, but species identification by this method was very difficult. All negative control samples gave negative results by $\mathrm{QBC}$. The average time required to process and examine the specimens by QBC was 9 minutes.

The Parasight ${ }^{\mathrm{TM}}$ F Dipstrips technique showed twenty-two positive and eight negative results for the infected blood samples and gave negative results with the ten neg- 
ative controls. The positive samples were confirmed to have P. falciparum after examination of thick blood films by light microscopy. Serum samples positive for the R.F. were also positive with Parasight-F. The average time required to process and examine the specimens by this method was 6 minutes (Table 1).

TABLE 1. Comparison between thick blood films, QBC technique, and Parasight-F Dipstrip.

\begin{tabular}{|l|c|c|c|c|c|c|c|}
\hline \multirow{2}{*}{ Group } & \multirow{2}{*}{ \# of Subjects } & \multicolumn{2}{c|}{ Blood Films } & \multicolumn{2}{c|}{ QBC } & \multicolumn{2}{c|}{ Dipstrip } \\
\cline { 3 - 9 } & & Positive & Negative & Positive & Negative & Positive & Negative \\
\hline Negative Control & 10 & 0 & 10 & 0 & 10 & 0 & 10 \\
\hline Infected Blood & 30 & 30 & 0 & 30 & 0 & 22 & 8 \\
\hline Serum Samples & 7 & - & - & - & - & 7 & - \\
\hline \multicolumn{2}{|l|}{ Average Time Required(Minutes) } & \multicolumn{2}{|c|}{19} & \multicolumn{2}{|c|}{9} & \multicolumn{2}{c|}{6} \\
\hline
\end{tabular}

\section{Discussion}

Malaria is one of the few acute parasitic infections that can be life-threatening and needs to be diagnosed as soon as possible to protect the patient from further complications. The definitive diagnosis of malaria is based on the demonstration of the parasites in the blood. Two types of blood films are used: (1) the thick blood film allows the examination of a larger amount of blood and is used as a screening procedure; (2) the thin blood film allows species identification of the parasite. Blood films are usually prepared when the patient is admitted and samples should be taken at intervals of 6 to 8 hours for at least 3 successive days before the film is signed out as negative ${ }^{[7]}$. Micrscopic examination of Romanowsky stained thick blood films is at present the most practical and reliable method for diagnosing malarial infections. When the parasitaemia is low, the technique can be time-consuming. Another limitation is it requires an experienced Microscopist and it is difficult to apply in the field. Parasite diagnosis by the QBC method is more sensitive than conventional blood film microscopy due to the comparatively larger volume of blood screened of parasitic infection ${ }^{[8]}$.

Additionally, the QBC method concentrates most of the parasites into the narrow zone that is optically examined. Also, QBC technique is more rapid in detecting malaria parasites and often is more sensitive when performed by well-trained technicians since it needs less technical skills than that needed to examine a thick blood film. However, the QBC method is unreliable for field studies. Other limitations are that it requires the purchasing of expensive equipment, the high cost of the diagnostic kit, and the difficulties in distinguishing between different Plasmodium species.

The Dipstrip immunoassay might be considered as the most efficient technique that can be used in survey studies for P. falciparum in endemic areas. However, this method can give false positive results if the sample contains high levels of R.F. as a cross reaction $^{[9]}$. Also, the Dipstrip method may be used to rapidly confirm the presence of $P$. falciparum diagnosed by other techniques. 
QBC is a qualitative screening method. Although parasites are detected by observing florescence of Acridine Orange (AO) within the tube, species identifications requires examination of routine stained blood films. Specimens may be submitted from patients with $P$. falciparum infections who do not yet have gametocytes in the blood. Consequently, a low-level parasitaemia with a delicate ring form might be missed without extensive oil immersion examination of the blood films (at least 200 to 300 oil immersion fields) ${ }^{[7]}$. Many studies have used immunodiagnostic procedures for the diagnosis of malaria; however, these procedures are not routinely performed in most laboratories. Species identification is very important because some stains have developed a partial resistance to quinine as well as required use of combination therapy. Certain strains of P. falciparum in South America and Southeast Asia was found to be resistant to treatment by some antimalarial drugs as chloroquine. Therefore, some of these drugs may not be useful for treatment until the causative species are exactly identified.

In conclusion, thick and thin blood films remain the methods of choice for diagnosing malarial infections. They are rapid and can be applied in any laboratory or in the field with minimum equipment.

\section{References}

[1] Kuate Defo B. Epidemiology and control of infant and early childhood malaria: a competing risks analysis. Int J Epidemiol 1995; 24(1): 204-217.

[2] Cheesbrough M. Medical Laboratory Manual for Tropical Countries, 2nd Edition. ELBS with Tropical Health Technology/Butterworth-Hheinemann. 1991; Vol. 1: 605.

[3] Howard RJ, Uni S, Aikawa M, Aley SB, Leech JH, Lew AM, Wellems TE, Rener J, Taylor DW. Secretion of a malaria histidine-rich protein (Pf HRPII) from Plasmodium falciparum-infected erythrocytes. J Cell Biol 1986; 103(4): 1269-1277.

[4] Rock EP, Marsh K, Saul AJ, Wellems TE, Taylor DW, Maloy WL, Howard RJ. Comparative analysis of the Plasmodium falciparum histidine-rich proteins (HRP-I, HRP-II, and HRP-III) in malaria parasites of diverse origin. Parasitology 1987; 95(Pt 2): 209-227.

[5] Parra ME, Evans CB, Taylor DW. Identification of Plasmodium falciparum histidine-rich protein 2 in the plasma of humans with malaria. J Clin Microbiol 1991; 29(8): 1629-1634.

[6] Rodriquez-del-Valle M, Quakyi IA, Amuesi J, Quaye JT, Nkrumah FK, Taylor DW. Detection of antigens and antibodies in the urine of humans with Plasmodium falciparum malaria. J Clin Microbiol 1991; 29(6): 1236-1242.

[7] Baron D, Gazin P, Rea D, Carneval C. Diagnostic Microbiology, 9th Edition. Bailey \& Scott's; Missouri. 1994: 955.

[8] Spielman A, Perrone JB, Teklehaimanot A, Balcha F, Wardlaw SC, Levine RA. Malaria diagnosis by direct observation of centrifuged samples of blood. Am J Trop Med Hyg 1988; 39(4): 337-342.

[9] Grobusch MP, Alpermann U, Schwenke S, Jelinek T, Warhurst DC. False-positive rapid tests for malaria in patients with rheumatoid factor. Lancet 1999; 353: 297. 


\section{مقارنة مابين أفلام الدم السميكة وطريقة الغلاف الأبيض \\ الكمية وطريقة الشرائح البلاستيكية لتشخيص مرض الملاريا}

\section{هيثم أحمد زكائي}

قسم برنامج التقنية الطبية ، كلية العلوم الطبية التطبيقية،

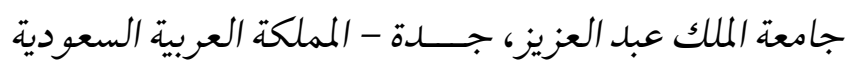

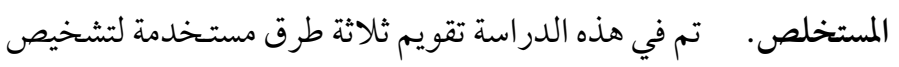

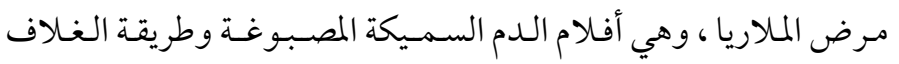

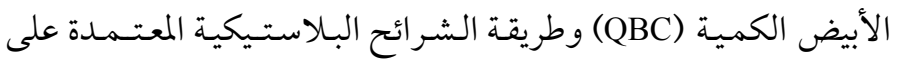

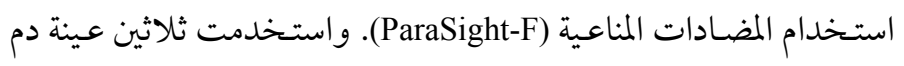

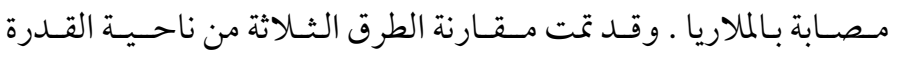

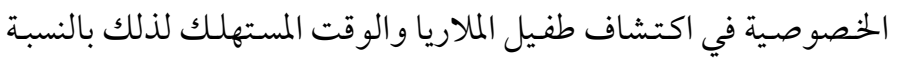

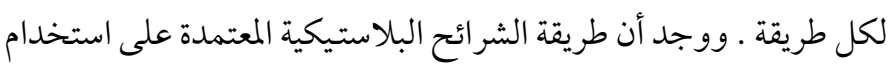

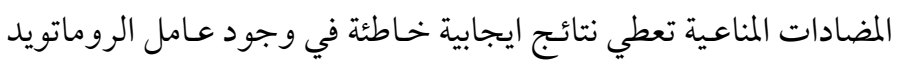

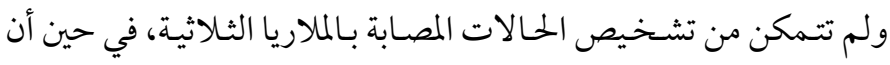

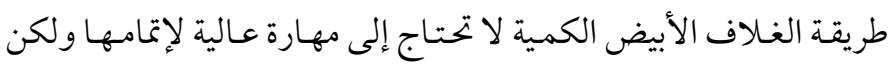

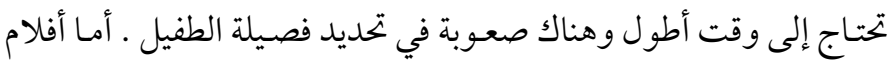

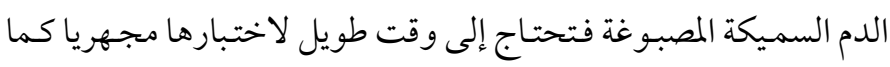

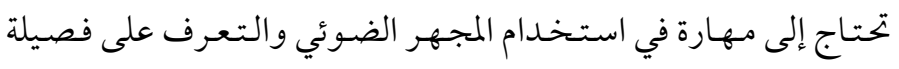

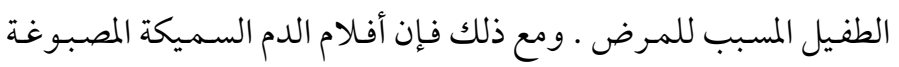

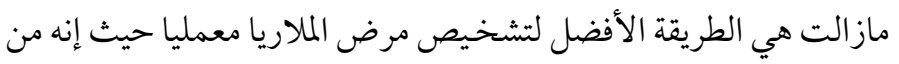

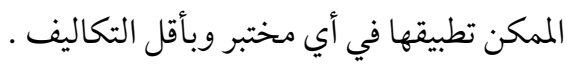

